\title{
SNHG17 promotes gastric cancer cell proliferation and invasion by suppressing RUNX3 via interacting with EZH2
}

\author{
Xinmei ZHANG ${ }^{1}$, Guofang FAN ${ }^{1}$, Jianghong ZHAN ${ }^{1}$, Zihua GUAN ${ }^{2 *}$
}

\begin{abstract}
Accumulating data has indicated that long noncoding RNAs (lncRNAs) play critical roles in multiple cancers including gastric cancer (GC). However, the functional role of SNHG17 in gastric cancer (GC) development and related biological mechanisms remain unknown. In the study, our results demonstrated that SNHG17 expression was higher in tumor tissues compared to adjacent normal tissues in 99 cases of patients with GC. Higher SNHG17 expression positively associated with lymph node metastasis, advanced TNM stage and predicted a worse prognosis in GC patients. Functionally, we showed that overexpression of SNHG17 promoted cell proliferation, cell colony formation and cell invasion in vitro. However, SNHG17 knockdown significantly inhibited cell proliferation and invasion of GC. Furthermore, we demonstrated that SNHG17 promoted cell proliferation and invasion by regulating RUNX3 via interacting with EZH2 in GC cells. In addition, overexpression of SNHG17 reversed the cell proliferation and invasion induced by SNHG17 in GC. Thus, these results indicated that SNHG17 could serve as a prognostic biomarker and target of GC treatment.
\end{abstract}

Keywords: gastric cancer; long noncoding RNAs; SNHG17; EZH2; RUNX3.

Practical Application: SNHG17 expression was higher in GC tissues and cells.

\section{Introduction}

Gastric cancer (GC) is one of the most common types of gastrointestinal cancers with an estimated 1,000,000 new cases per year occur (Jemal et al., 2011). Due to local and systemic metastasis of this disease at advanced stage, the 5-year survival rate remain poor (Bickenbach and Strong, 2012; Thiel and Ristimäki, 2012). Accumulating studies have implied that the pathogenesis of gastric cancer closely associated with the activity of oncogenes or tumor suppressors (Carcas, 2014). To explore underlying molecular mechanisms could provide potential value for GC treatment.

Long noncoding RNAs have been found to show significant roles in a wide variety of biological effects including cell proliferation, cell cycle, cell stress responses, cell immunity, cell differentiation and cell apoptosis in tumor progression (Batista and Chang, 2013; Guo et al., 2015). Long non-coding RNA AK096174 promotes cell proliferation and invasion in gastric cancer by regulating WDR66 expression (Zhang et al., 2018b). Long non-coding RNA SNHG14 contributes to gastric cancer development through targeting miR-145/SOX9 axis (Liu et al., 2018). Long non-coding RNA AFAP1-antisense RNA 1 promotes the proliferation, migration and invasion of gastric cancer cells and is associated with poor patient survival (Zhao et al., 2018). Previous studies have demonstrated that dysregulation of SHNG17 in colorectal cancer, Ma et al.showed that long noncoding RNA SNHG17 is a poor prognostic factor and promotes cell proliferation by epigenetically silencing P57 in colorectal cancer (Ma et al., 2017). However, the role of SNHG17 in GC development remains unknown.

In the study, we showed that SNHG17 expression was higher in GC tissues compared to adjacent normal tissues and predicted a worse prognosis. Functionally, we showed that overexpression of SNHG17 promoted cell proliferation and invasion in vitro. Furthermore, we demonstrated that SNHG17 promoted cell proliferation and invasion by regulating RUNX3 in GC cells. Thus, these results indicated that SNHG17 could serve as a target of GC treatment.

\section{Materials and methods}

\subsection{Tissue samples and cell culture}

99 cases of GC tissues and matched adjacent normal tissue samples were collected from patients including 59 males and 40 females who received surgical resection between August 2012 and July 2015. The age range was between 35 and 76 years old, and the median age was 50 years. All tissue samples were snap-frozen in liquid nitrogen immediately following resection and stored at $-80^{\circ} \mathrm{C}$ until RNA extraction. No local or systemic treatment had been applied to the patients before surgery. All protocols were approved by the People Hospital of Changshan County and written informed consent was obtained from the patients in the study. 
Human GC cell lines including MKN-45, BGC-823, MGC803 and SGC-7901 cells and GES-1 were purchased from Biochemistry and Cell Biology at the Chinese Academy of Sciences (Shanghai, China). Cells were cultured in Dulbecco's modified Eagle's medium (DMEM; Invitrogen; Waltham, MA, USA) containing $10 \%$ fetal bovine serum (FBS, Gibco, Grand Island, NY, USA), $1 \%$ penicillin and $1 \%$ streptomycin (Gibco; Thermo Fisher Scientific, Inc.). The cells were incubated at $37^{\circ} \mathrm{C}$ in a humidified atmosphere with $5 \% \mathrm{CO}_{2}$.

\subsection{RNA extraction and quantitative reverse transcription polymerase chain reaction ( $R T-q P C R)$ analysis}

Total RNA was isolated from tissue and cell samples using TRIzol reagent (Takara Biotechnology Co., Ltd., Dalian, China) according to the manufacturers' protocols. The cDNA was reversed transcription from RNA samples using Prime Script ${ }^{\mathrm{mt}}$ RT Master Mix (Takara Biotechnology Co., Ltd., Dalian, China). The qRT-PCR reaction was performed using Power SYBR Green PCR Master mix (Applied Biosystems, Thermo Fisher Scientific, Inc.) according to the manufacturer's protocol. The PCR primer sequences for SNHG17 were as follows: SNHG17 forwards: 5'-TGCTTGTAAGGCAGGGTCTC-3', and SNHG17 reverse: 5'-ACAGCCACTGAAAGCATGTG-3'. Relative mRNA expression was calculated using the comparative cycle threshold method (value of $2^{-\Delta \Delta \mathrm{Ct}}$ ) and the GAPDH mRNA expression was as an endogenous control. Each sample was analyzed in triplicate.

\subsection{RNA interference and vectors}

Two small interfering RNAs (siRNAs) specifically targeting human SNHG17 were purchased from Gene Pharma (Shanghai, China). si-SNHG17-1: 5'-AAACGAGCGTAGCTTCCTT3';si-SNHG17-2:5'-GCAGTGTCTCGTCCTCTTT-3'.The siRNAs were transfected into MKN-45 and SGC-7901 cells using the RNAiMAX reagent (Life Technologies) according to the manufacturer's instructions. Vector pcDNA-SNHG17 and pcDNA-RUNX3 were constructed and purchased from Genechem (Shanghai, China). The plasmids were transfected into cells via using the lipofectamine 2000 (Life Technologies).

\subsection{Cell proliferation and cell colony assays}

Cell proliferation was determined using CCK8 assays. Transfected MKN-45 and SGC-7901 cells were seeded in 96-well plates at a density of 3000 cells/well, and cultured for $0,1,2$, 3 and 4 days. At these indicated times, CCK8 assays were carried out. Briefly, $10 \mu \mathrm{L}$ CCK8 solution (Sigma-Aldrich, Germany) was added into each well and incubated for additional $2 \mathrm{~h}$ at $37^{\circ} \mathrm{C}$ with $5 \% \mathrm{CO}_{2}$. The absorbance at $450 \mathrm{~nm}$ was detected using a microplate reader (iMark ${ }^{\text {min}}$; Bio-Rad Laboratories, Inc., Hercules, CA, USA).

For cell colony formation, transfected $\mathrm{MKN}-45$ and SGC-7901 cells were seeded in 12-well plates at a density of 500 cells/well, after cell transfection at 7 days, cell were fixed in $100 \%$ methanol for $15 \mathrm{~min}$, stained with $0.1 \%$ crystal violet for $15 \mathrm{~min}$, and photographed under a light microscope (Olympus Corporation, Tokyo, Japan).

\subsection{Cell invasion assay}

Cell invasion assays were performed using Transwell chambers ( $8 \mathrm{~mm}$ pores; Costar, Corning Incorporated, NY USA) precoated with Matrigel solution (BD Biosciences, San Jose, CA, USA). After transfection for $48 \mathrm{~h}, 5 \times 10^{5}$ cells cultured with FBS-free DMEM medium were added into the upper chamber. In the lower chamber, $500 \mu \mathrm{L}$ DMEM with 20\% FBS was added. After cell transfection at $48 \mathrm{~h}$, cells in the upper surface of the membrane were removed with cotton swab. Cells in the membrane surface were fixed with $100 \%$ methanol for $20 \mathrm{~min}$, stained with $0.1 \%$ crystal violet for $15 \mathrm{~min}$, and counted under a light microscope (magnification 200×, Olympus Corporation, Tokyo, Japan) in five randomized fields.

\subsection{Western blot analysis}

Total protein was extracted from the cells using RIPA lysis buffer (Beyotime, China) according to the manufacturers' protocols. The protein concentration was quantified using the BCA kit (Beyotime, China) according to the recommended protocol. Equal amount of protein sample was separated on 10$12 \%$ SDS-PAGE gel and then transferred to PVDF membrane. The membrane was then incubated with primary antibody against RUNX3 (1:1000, Cell Signaling Technology, Inc., Danvers, MA, USA) and GAPDH (1:1000, Cell Signaling Technology, Inc., Danvers, MA, USA) at $4{ }^{\circ} \mathrm{C}$ overnight. Then, the membranes were blocked using $5 \%$ fat-free milk. After that, the membrane was incubated with horseradish peroxidase (HRP) conjugated secondary antibody (1:1000, Cell Signaling Technology, Inc., Danvers, MA, USA) for $2 \mathrm{~h}$ at room temperature. The Chemi Doc $^{\text {mm }}$ XRS+ system (Bio-Rad Laboratories, Inc., Hercules, CA, USA) was performed to visualize the bands. The results were quantified with Quantity One software (Bio-Rad, Hercules, CA, USA). The protein expression of GAPDH was used as an internal control.

\subsection{RNA Immunoprecipitation (RIP) assays}

Magna RIP ${ }^{\mathrm{m}}$ RNA-Binding Protein Immunoprecipitation Kit (Millipore) was applied to perform RIP experiments on the basis of the manufacture's manual. Antibody for EZH2 RIP assay was purchased from Cell Signaling Technology. IgG antibody (Cell Signaling Technology) was used as a negative control. The co-precipitated RNAs were purified and analyzed by qRTPCR analysis.

\subsection{Statistical analysis}

All statistical analysis was performed using SPSS 17.0 software (SPSS Inc., Chicago, IL, USA) and the results were shown as the mean \pm standard deviation (SD) from three independent repeated experiments. Statistical significance was analyzed using two-tailed Student's $t$-test. More than two groups were analyzed using a one-way analysis of variance followed by a Newman-Keuls test. $\mathrm{P}<0.05$ was considered to indicate a statically significant difference. 


\section{Results}

\subsection{Increased expression of SNHG17 positively associates with poor survival overall survival in patients with GC}

To investigate SNHG17 expression in GC, 99 pairs of GC tissues and adjacent normal tissues were examined using qRT-PCR analysis. The qRT-PCR results showed that SNHG17 expression was significantly increased in GC tissues compared with adjacent normal tissues $(\mathrm{P}<0.05$; Figure 1A). SNHG17 expression was divided into higher SNHG17 expression and lower SNHG17 expression group according to mean expression level. Further analysis revealed that SHNG17 expression positively associated with lymph node metastasis and advanced TNM stage $(\mathrm{P}<0.05$, Table 1$)$. Additionally, survival plots analysis indicated that patients with GC with higher expression of SNHG17 exhibited a poor survival rate compared with those

\section{A}

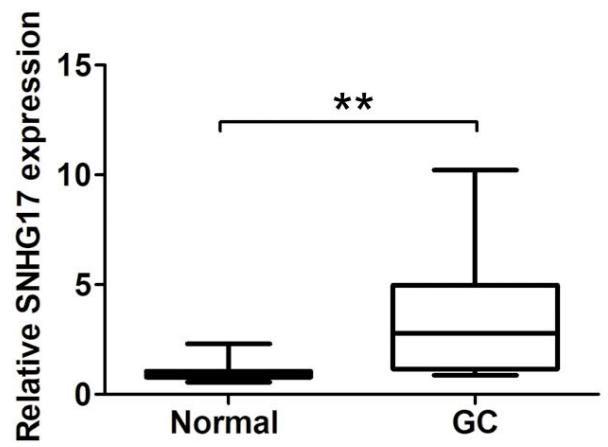

C

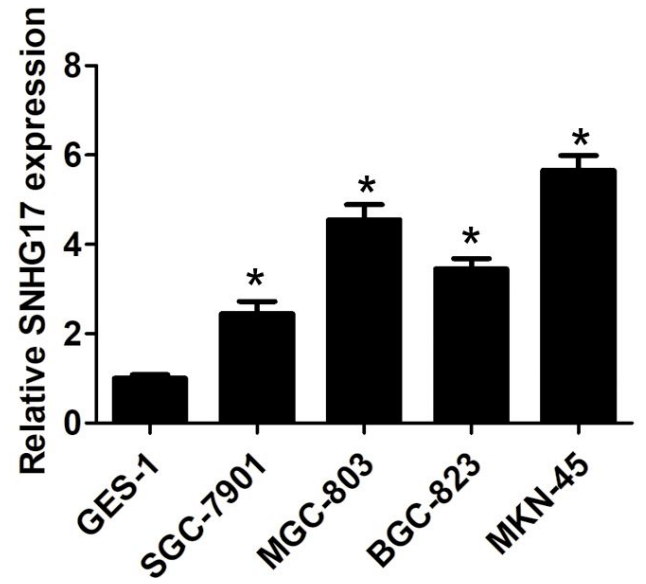

\section{A}

with lower expression of SNHG17 (Figure 1B). These results suggested that SNHG17 expression may serve as a novel biomarker for the prognosis of GC.

\subsection{Increased expression of SNHG17 promotes cell proliferation and invasion in GC}

To understand the role of SNHG17 expression in tumor progression of GC, we first analyzed the SNHG17 expression in several human GC cell lines including MKN-45 BGC-823, MGC-803 and SGC-7901 and the normal gastric epithelial cell line GES-1. The qRT-PCR results indicated that SNHG17 expression was significantly increased in GC cells compared to normal gastric epithelial cell line GES-1 (Figure 1C). Next, we performed gain function and loss gain function assay in SGC-7901 and MKN-45 cells, respectively (Figure 2A-B). The cell proliferation

B
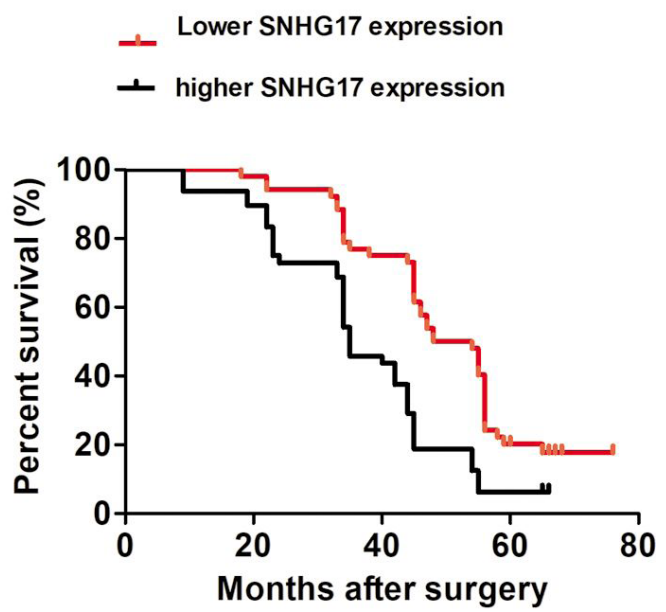

Figure 1. SNHG17 is highly expressed in GC tissues and cells. (A) QRT-PCR analysis of SNHG17 expression in 99 paired GC tissues and their adjacent normal tissues. SNHG17 expression was normalized to GAPDH expression; (B) Kaplan-Meier overall survival analysis of the association between SNHG17 expression and GC patient survival ability; (C) QRT-PCR analysis of SNHG17 expression in cell lines BGC823, SGC7901, MGC803, MKN-45, and GSE-1. SNHG17 expression was normalized to that of the GAPDH expression level. ${ }^{* *} \mathrm{P}<0.01$, ${ }^{*} \mathrm{P}<0.05$; OS, overall survival. 
Table 1. The association of SNHG17 expression with clinicopathologic factors in 99 GC patients.

\begin{tabular}{|c|c|c|c|c|}
\hline \multirow[b]{2}{*}{$\begin{array}{c}\text { Clinicopathologic } \\
\text { factors }\end{array}$} & \multicolumn{4}{|c|}{ SNHG17 expression } \\
\hline & $\begin{array}{c}\text { Total } \\
(\mathrm{n}=99)\end{array}$ & $\begin{array}{l}\text { Lower } \\
(\mathrm{n}=47)\end{array}$ & $\begin{array}{l}\text { Higher } \\
(\mathrm{n}=52)\end{array}$ & $P$ value \\
\hline Age (years) & & & & 0.154 \\
\hline$\leq 60$ & 35 & 20 & 15 & \\
\hline$>60$ & 64 & 27 & 37 & \\
\hline Sex & & & & 0.414 \\
\hline Male & 59 & 30 & 29 & \\
\hline Female & 40 & 17 & 23 & \\
\hline Tumor size & & & & 0.096 \\
\hline$<3 \mathrm{~cm}$ & 44 & 25 & 19 & \\
\hline$\geq 3 \mathrm{~cm}$ & 55 & 22 & 33 & \\
\hline Grade & & & & 0.997 \\
\hline Highly & 40 & 19 & 21 & \\
\hline Moderately & 34 & 16 & 18 & \\
\hline Lowly & 25 & 12 & 13 & \\
\hline $\begin{array}{l}\text { Lymph node } \\
\text { metastasis }\end{array}$ & & & & $0.001^{*}$ \\
\hline Positively & 49 & 15 & 34 & \\
\hline Negatively & 50 & 32 & 18 & \\
\hline TNM stage & & & & $0.014^{*}$ \\
\hline I-II & 59 & 34 & 25 & \\
\hline III-IV & 40 & 13 & 27 & \\
\hline
\end{tabular}

A

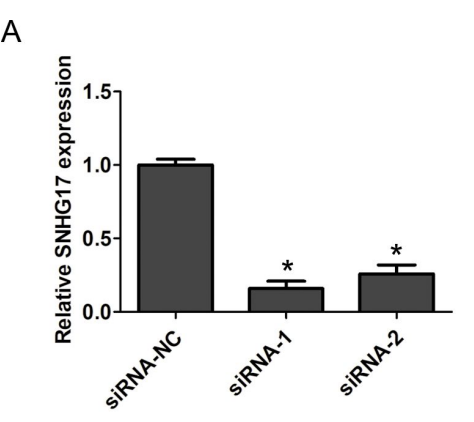

D

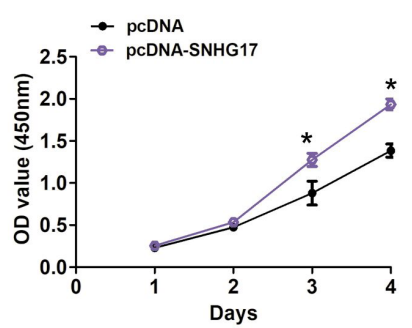

capacity of the above-mentioned cell lines was analyzed, we found that MKN-45 cell line with si-SHNG17-1 had reduced cell proliferation rate compared with negative control (Figure 2C). Conversely, SGC-7901 cell line with pcDNA-SHNG17 had increased cell proliferation rate compared with negative control (Figure 2D). Furthermore, we found that MKN-45 cell line with si-SHNG17-1 had reduced cell colony formation ability compared with negative control (Figure 2E). Conversely, SGC-7901 cell line with pcDNA-SHNG17 had increased cell proliferation rate compared with negative control (Figure 2F).

Moreover, we found that knockdown of SNHG17 reduced the cell invasion ability compared to negative control group, however, overexpression of SNHG17 enhanced the cell invasion ability compared to negative control group 1 (Figure 3A-D). Thus, these results indicated that SNHG17 could promoted cell proliferation and invasion in GC.

\subsection{Increased expression of SNHG17 suppresses RUNX3 by interacting $\mathrm{EZH} 2$}

Studies have found that about $20 \%$ of human lncRNAs recruit $\mathrm{EZH} 2$ to specific genome sites to silence gene expression (Deng et al., 2017). Long non-coding RNA SNHG17 is an poorly prognostic factor and promotes cell proliferation by epigenetically silencing P57 in colorectal cancer (Ma et al., 2017). RUNX3, acts as a tumor suppressor, has been reported to
B

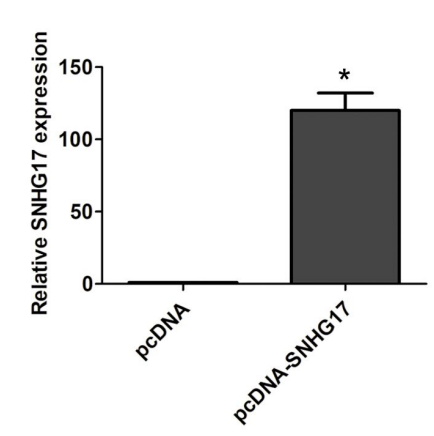

$E$

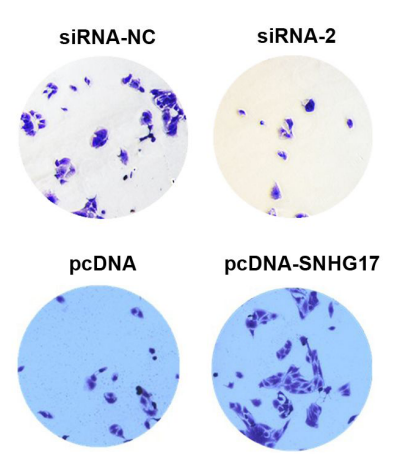

C

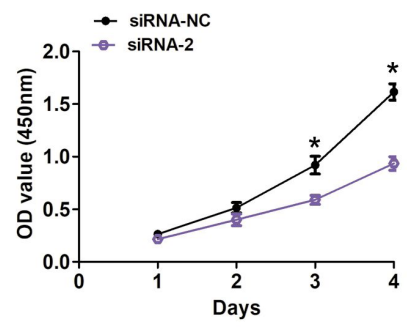

$\mathrm{F}$

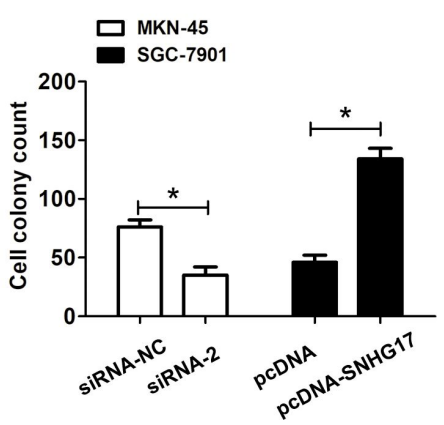

Figure 2. SNHG17 promotes cell proliferation in vitro. (A) QRT-PCR analysis of SNHG17 expression in cell lines MKN-45 transfected with SNHG17-1, SNHG17-2 or siRNA-NC; (B) QRT-PCR analysis of SNHG17 expression in cell lines MKN-45 transfected with pcDNA plasmid or pcDNA-SNHG17; (C) Growth curves for MKN-45 cells after transfection with SNHG17 siRNA-2 or the negative control were determined by CCK8 assays; (D) Growth curves for SGC-7901 cells after transfection with pcDNA or pcDNA-SNHG17 were determined by CCK8 assays; (E) Colony formation assay and cell colony number were showed in MKN-45 cells after transfection with SNHG17 siRNA-2 or the negative control; (F) colony formation assay and cell colony number were showed in SGC-7901 cells after transfection with pcDNA or pcDNA-SNHG17 were determined by colony formation assay. ${ }^{*} \mathrm{P}<0.05$. 
inhibit the proliferation and metastasis of gastric cancer through regulating miR-182/HOXA9 (Yu et al., 2017). Thus, we speculated SNHG17 whether could regulate RUNX3 expression through EZH2 in GC. To prove this hypothesis, we examined the effects of SNHG17 knockdown on RUNX3 expression in GC cells. The results demonstrated that RUNX3 mRNA expression was evidently upregulated by transfecting with si-SNHG17 in MKN45 cells (Figure 4A-B). However, RUNX3 mRNA expression was evidently downregulated by transfecting with pcDNA-SNHG17 in SGC-7901 cells (Figure 4C-D). Furthermore, the results showed that SNHG17 is distributed in both the cytoplasm and nucleus in MKN-45 and SGC-7901 (Figure 5A-B). Subsequently, we found that SNHG17 was we probed the interaction possibility of SNHG17 and EZH2 using RIP assay. RIP assays showed that SNHG17 level was greatly enriched by EZH2 antibody (Figure 5CD). Together, these results indicated that SHNG17 could interact with EZH2 in GC cells.

\subsection{Overexpression of RUNX3 reverses cell proliferation and invasion induced by SNHG17 in GC}

To investigate the functional effects of RUNX3 in GC progression, we constructed RUNX3-overexpression plasmid (pcDNA-RUNX3) and transfected it into SGC-7901 cells. The qRTPCR and western analysis confirmed that RUNX3 expression was significantly upregulated after transfection with pcDNARUNX3 (Figure 6A-B). CCK-8 experiments showed that enforced expression of RUNX3 resulted in a decrease of cell proliferation in SGC-7901, but was reversed by co-transfection with pcDNARUNX3+si-SNHG17 (Figure 6C). Cell colony formation demonstrated that enforced expression of RUNX3 resulted in a decrease of cell proliferation in SGC-7901, but was reversed by co-transfection with pcDNA-RUNX3+si-SNHG17 (Figure 6D). Transwell invasion assay found that the cell invasive ability of SGC-7901 cells was substantially abated after introduction with pcDNA-RUNX3, but was reversed by cotransfection with si-SNHG17 (Figure 6E). Thus, these results indicated

\section{A}

B

SIRNA-NC

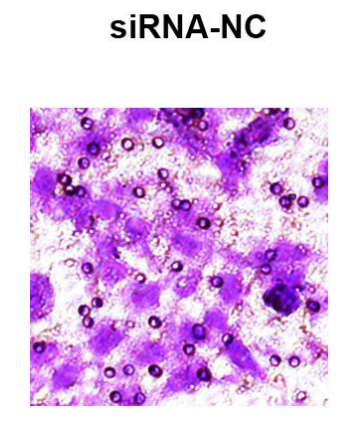

SiRNA-2

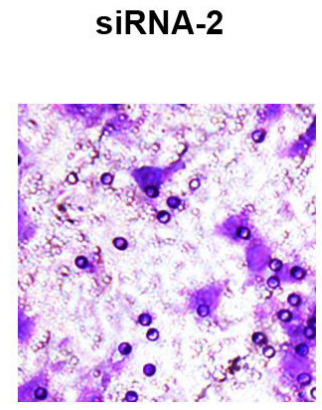

MKN-45
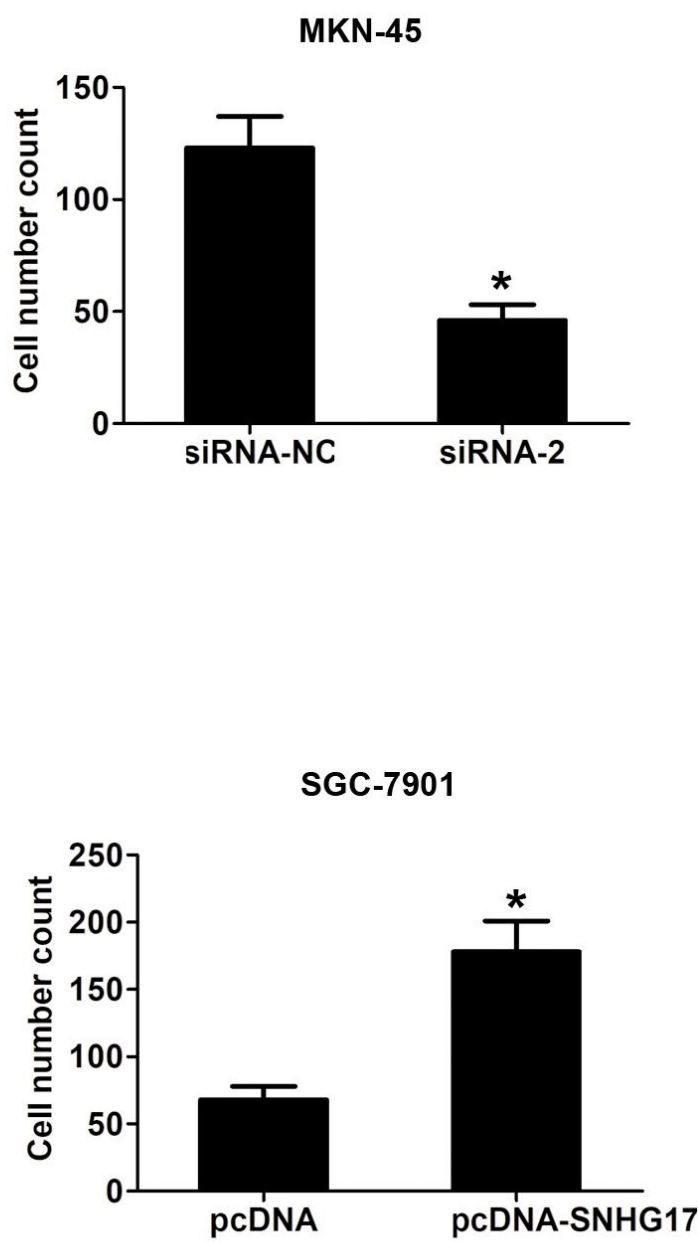

\section{SGC-7901}

Figure 3. SNHG17 promotes cell invasion in vitro. (A-B) Cell invasion and invasive cell number were showed for MKN-45 cells after transfection with SNHG17 siRNA-2 or the negative control; (C-D) Cell invasion and invasive cell number for SGC-7901 cells after transfection with pcDNA or pcDNA-SNHG17. ${ }^{\star} \mathrm{P}<0.05$. 
A

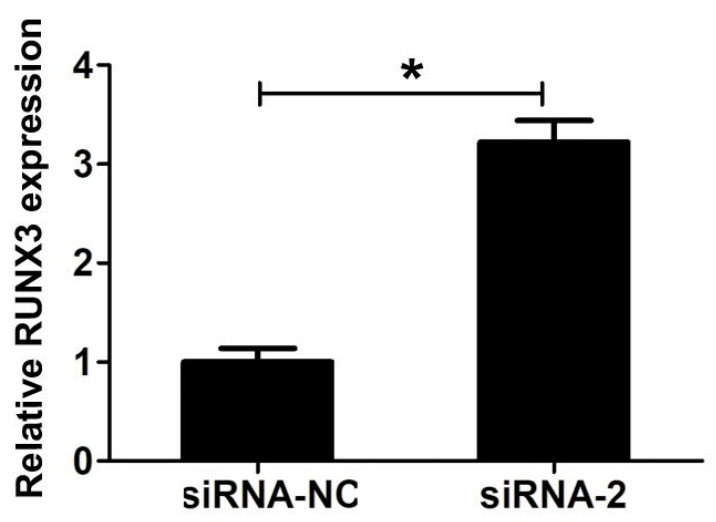

MKN-45
MKN-45

SIRNA-NC SIRNA-2

RUNX3

GAPDH
C

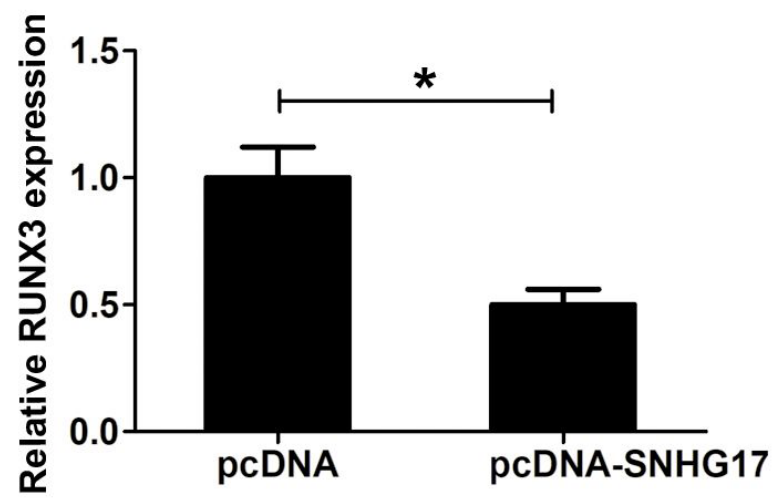

SGC-7901

pcDNA pcDNA-SNHG17

\section{RUNX3}

GAPDH

SGC-7901

Figure 4. SNHG17 regulated RUNX3 expression in GC cells. (A-B) The RUNX3 mRNA and protein expression were detected after SNHG17 knockdown in MKN-45 cells compared to siRNA-NC by using qRT-PCR or western blot analysis; (C-D) The RUNX3 mRNA and protein expression were detected after SNHG17 overexpression in SGC-7901 cells compared to pcDNA vector group by using qRT-PCR or western blot analysis.

that overexpression of RUNX3 reversed cell proliferation and invasion induced by SNHG17 in GC

\section{Discussion}

Recently, increasing studies have linked dysregulation of lncRNAs to diverse human diseases especially in tumorigenesis. Some evidence has revealed that lncRNAs could regulate cell proliferation, migration, invasion and metastasis in GC (Zhang and $\mathrm{Du}, 2016)$. Long noncoding AFAP1-antisense RNA 1 is upregulated and promotes tumorigenesis in gastric cancer (Ye et al., 2018). LncRNA-MEG3 inhibits proliferation and metastasis by regulating miRNA-21 in gastric cancer (Dan et al., 2018). Long non-coding RNA UFC1 promotes gastric cancer progression by regulating miR-498/Lin28b (Zhang et al., 2018a). However, the functional role of SNHG17 in gastric cancer (GC) development and related biological mechanisms remain unknown.

In the study, our results showed that SNHG17 expression was higher in GC tissues compared to adjacent normal tissues. Furthermore, we found that higher SNHG17 expression positively associated with lymph node metastasis, advanced TNM stage and predicted a worse prognosis in GC patients. Functionally, we showed that overexpression of SNHG17 promoted cell proliferation, cell colony formation and cell invasion in vitro. However, SNHG17 knockdown significantly inhibited cell proliferation and invasion of GC. These results indicated that SNHG17 play crucial role in GC progression.

Furthermore, we demonstrated that SNHG17 promoted cell proliferation and invasion by regulating RUNX3 via in interacting 
A

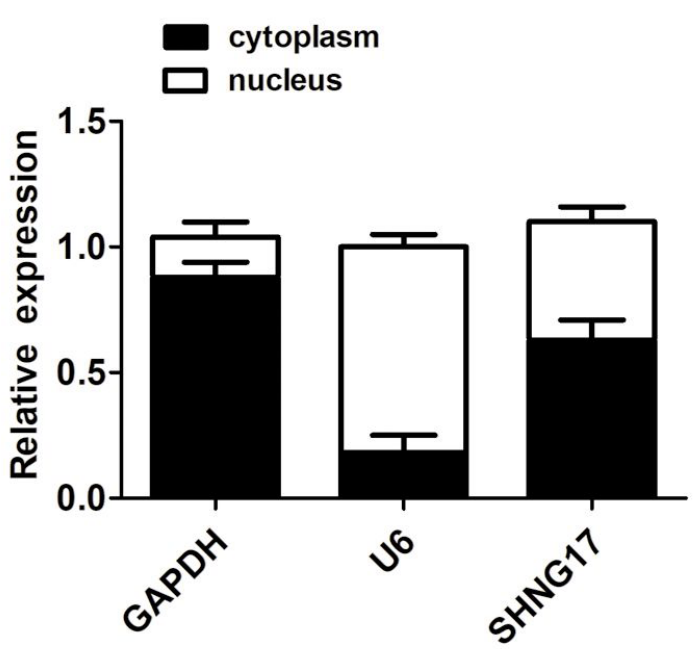

C
艺

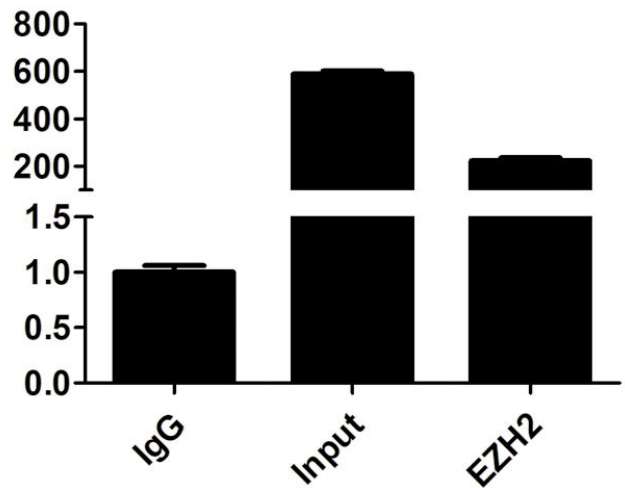

B
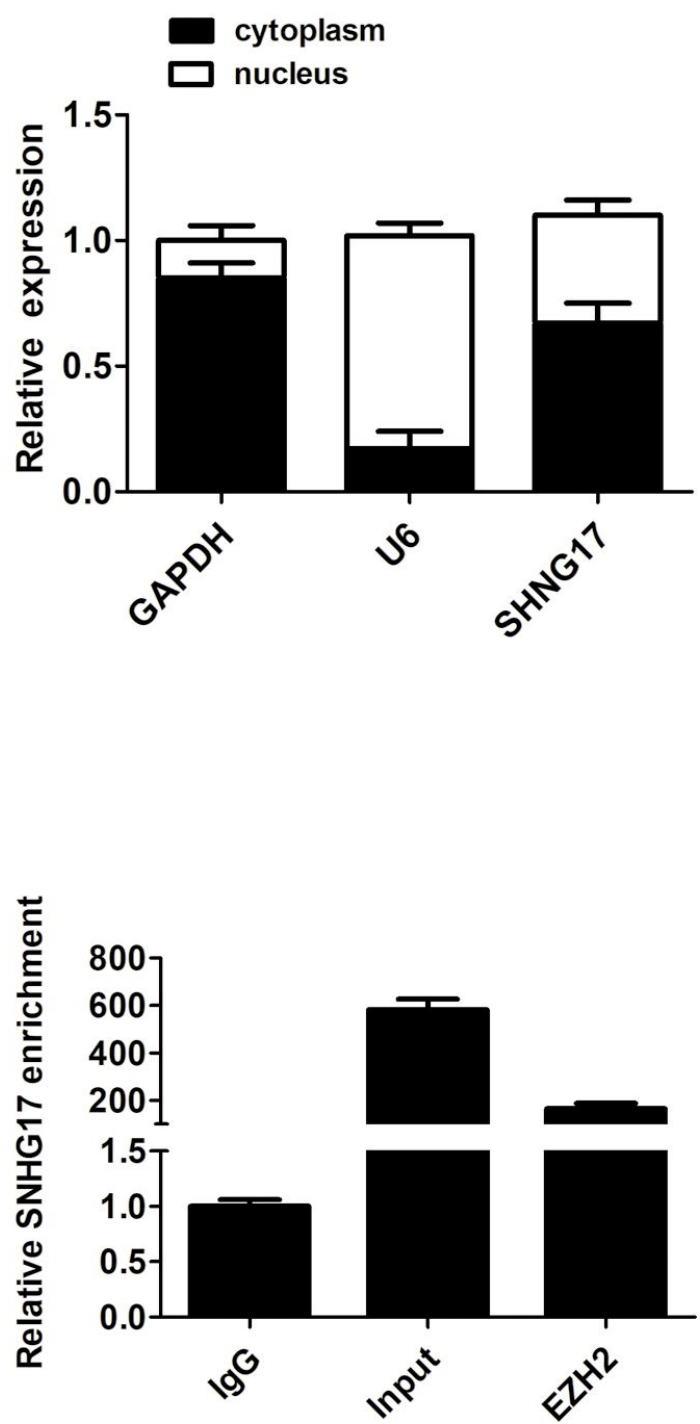

Figure 5. SNHG17 could bind to EZH2 in GC cells. (A-B) QRT-PCR detection of the percentage of SNHG17, U1, and GAPDH in the cytoplasm and nuclear fractions of MKN-45 and SGC-7901 cells. GAPDH and U1 were used as cytoplasmic and nuclear localization markers, respectively; (C-D) SNHG17 RNA levels in immunoprecipitates were determined by qRT-PCR. SNHG17 RNA expression levels are presented as fold enrichment values relative to IgG immunoprecipitates.

with EZH2 in GC cells. Overexpression of SNHG17 reversed the cell proliferation and invasion induced by SNHG17 in GC. In previous study, lncRNAs have been found to bind to $\mathrm{EZH} 2$ proteins regulates target gene promoters, thus contributes to biological behaviors in GC. Such as, long intergenic noncoding RNA 00152 promotes tumor cell cycle progression by binding to EZH2 and repressing p15 and p21 in gastric cancer (Chen et al., 2016). Long noncoding RNA HOXA-AS2 promotes gastric cancer proliferation by epigenetically silencing P21/ PLK3/DDIT3 expression (Xie et al., 2015). Long noncoding RNA PVT1 indicates a poor prognosis of gastric cancer and promotes cell proliferation through epigenetically regulating $\mathrm{p} 15$ and p16 (Kong et al., 2015). Long noncoding RNA LINC00673 is activated by SP1 and exerts oncogenic properties by interacting with LSD1 and EZH2 in gastric cancer (Huang et al., 2017).
Here, our results indicated that SNHG17 could promote cell proliferation and invasion by regulating RUNX3 expression.

One limitation in this study was that the food intake of the patients was not regularly recorded. It has been reported that food such as cheddar cheeses, synbiotic sheep milk and fermented bovine colostrum, have certain effect on the regulation of the biological activities (Balthazar et al., 2021; Gaspar-Pintiliescu et al., 2020; Rafiq et al., 2020), which may also affect the gastric cancer cell proliferation and invasion.

In conclusion, our results indicated that SNHG17 expression was higher in GC tissues and cells. Higher SNHG17 expression positively associated with a worse prognosis in GC patients. Functionally, we showed that overexpression of SNHG17 promoted cell proliferation and cell invasion in vitro by regulating 
A

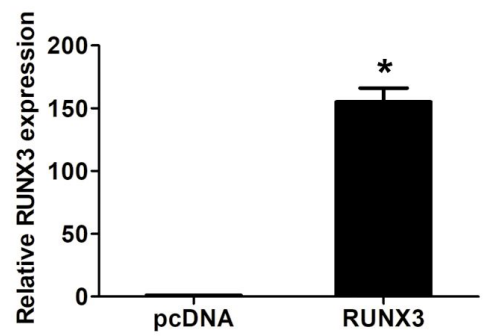

D

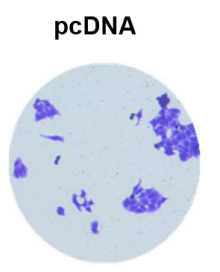

B

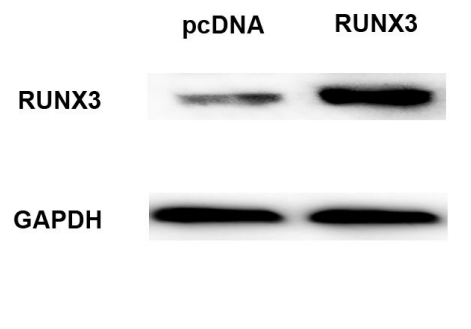

C

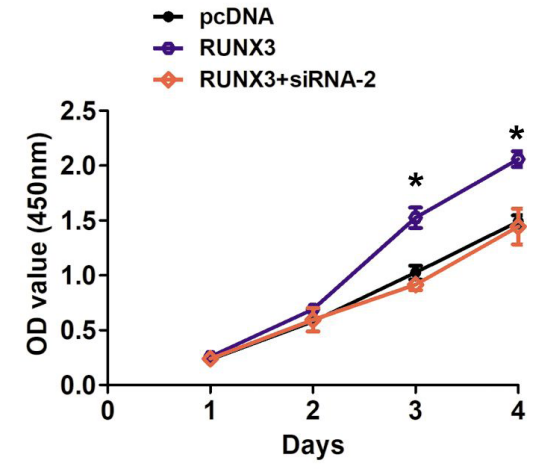

E

RUNX3+sIRNA-2

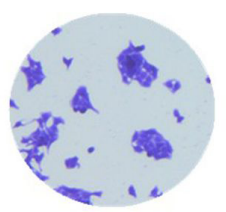

RUNX3

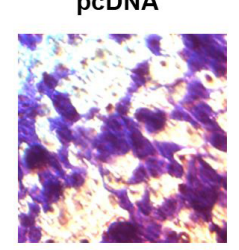

RUNX3+siRNA-2

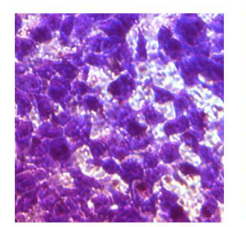

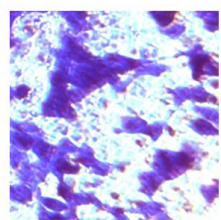
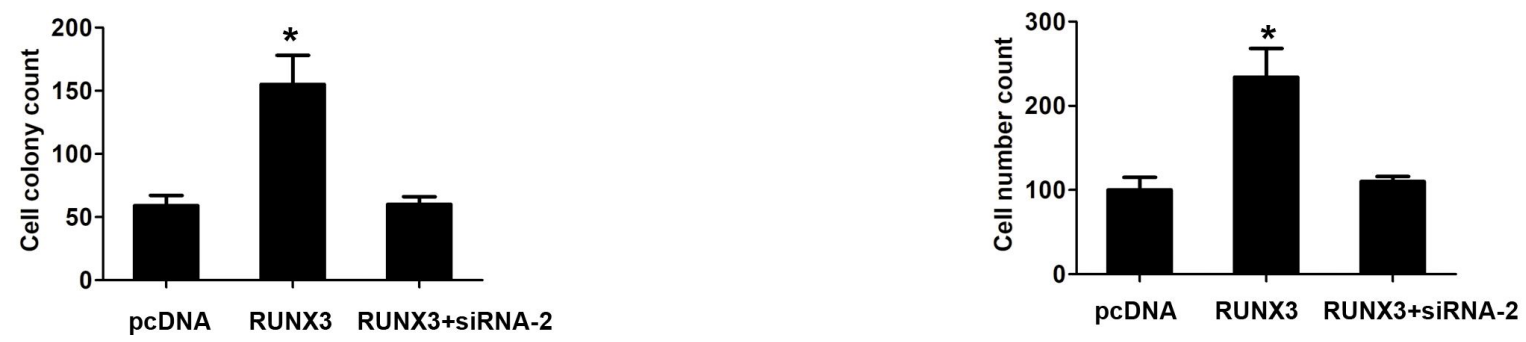

Figure 6. Overexpression of RUNX3 reverses cell proliferation and invasion induced by SNHG17 in GC. (A-B) The expression of RUNX3 was detected after SGC-7901 cells were transfected with pcDNA vector and pcDNA-RUNX3 plasmid by qRT-PCR or western blot analysis; (C) Cell proliferation ability was shown after SGC-7901 cells were transfected with pcDNA vector, pcDNA-RUNX3 and pcDNA-RUNX3 and siRNA-SNHG17 by CCK8 assays; (D) Cell colony formation ability was shown after SGC-7901 cells were transfected with pcDNA vector, pcDNA-RUNX3 and pcDNA-RUNX3 and siRNA-SNHG17; (E) Cell invasion ability was shown after SGC-7901 cells were transfected with pcDNA vector, pcDNA-RUNX3 and pcDNA-RUNX3 and siRNA-SNHG17 by transwell invasion assay. ${ }^{*} \mathrm{P}<0.05$.

RUNX3 expression. Thus, to further investigate the role of SNHG17 in GC could help find novel ways to therapy of GC patients.

\section{Conflict of interest}

The author declare that they have no conflict of interest.

\section{Funding}

The authors declare that no financial support was received.

\section{References}

Balthazar, C. F., Moura, N. A., Romualdo, G. R., Rocha, R. S., Pimentel, T. C., Esmerino, E. A., Freitas, M. Q., Santillo, A., Silva, M. C., Barbisan, L. F., Cruz, A. G., \& Albenzio, M. (2021). Synbiotic sheep milk ice cream reduces chemically induced mouse colon carcinogenesis. Journal of Dairy Science, 104(7), 7406-7414. http:// dx.doi.org/10.3168/jds.2020-19979. PMid:33934866.

Batista, P. J., \& Chang, H. Y. (2013). Long noncoding RNAs: cellular address codes in development and disease. Cell, 152(6), 1298-1307. http://dx.doi.org/10.1016/j.cell.2013.02.012. PMid:23498938.

Bickenbach, K., \& Strong, V. E. (2012). Comparisons of gastric cancer treatments: East vs. West. Journal of Gastric Cancer, 12(2), 55-62. http://dx.doi.org/10.5230/jgc.2012.12.2.55. PMid:22792517.

Carcas, L. P. (2014). Gastric cancer review. Journal of Carcinogenesis, 13(1), 14. http://dx.doi.org/10.4103/1477-3163.146506. PMid:25589897.

Chen, W. M., Huang, M. D., Sun, D. P., Kong, R., Xu, T. P., Xia, R., Zhang, E. B., \& Shu, Y. Q. (2016). Long intergenic non-coding RNA 00152 promotes tumor cell cycle progression by binding to EZH2 and repressing p15 and p21 in gastric cancer. Oncotarget, 7(9), 97739787. http://dx.doi.org/10.18632/oncotarget.6949. PMid:26799422.

Dan, J., Wang, J., Wang, Y., Zhu, M., Yang, X., Peng, Z., Jiang, H., \& Chen, L. (2018). LncRNA-MEG3 inhibits proliferation and metastasis by regulating miRNA-21 in gastric cancer. Biomedicine 
and Pharmacotherapy, 99, 931-938. http://dx.doi.org/10.1016/j. biopha.2018.01.164. PMid:29710493.

Deng, X., Zhao, Y., Wu, X., \& Song, G. (2017). Upregulation of CCAT2 promotes cell proliferation by repressing the P15 in breast cancer. Biomedicine and Pharmacotherapy, 91, 1160-1166. http://dx.doi. org/10.1016/j.biopha.2017.05.030. PMid:28531944.

Gaspar-Pintiliescu, A., Oancea, A., Cotarlet, M., Vasile, A. M., Bahrim, G. E., Shaposhnikov, S., Craciunescu, O., \& Oprita, E. I. (2020). Angiotensin-converting enzyme inhibition, antioxidant activity and cytotoxicity of bioactive peptides from fermented bovine colostrum. International Journal of Dairy Technology, 73(1), 108-116. http:// dx.doi.org/10.1111/1471-0307.12659.

Guo, L. L., Song, C. H., Wang, P., Dai, L. P., Zhang, J. Y., \& Wang, K. J. (2015). Competing endogenous RNA networks and gastric cancer. World Journal of Gastroenterology, 21(41), 11680-11687. http:// dx.doi.org/10.3748/wjg.v21.i41.11680. PMid:26556995.

Huang, M., Hou, J., Wang, Y., Xie, M., Wei, C., Nie, F., Wang, Z., \& Sun, M. (2017). Long noncoding RNA LINC00673 is activated by SP1 and exerts oncogenic properties by interacting with LSD1 and EZH2 in gastric cancer. Molecular Therapy, 25(4), 1014-1026. http://dx.doi. org/10.1016/j.ymthe.2017.01.017. PMid:28214253.

Jemal, A., Bray, F., Center, M. M., Ferlay, J., Ward, E., \& Forman, D. (2011). Global cancer statistics. CA: a Cancer Journal for Clinicians, 61(2), 69-90. http://dx.doi.org/10.3322/caac.20107. PMid:21296855.

Kong, R., Zhang, E. B., Yin, D. D., You, L. H., Xu, T. P., Chen, W. M., Xia, R., Wan, L., Sun, M., Wang, Z. X., De, W., \& Zhang, Z. H. (2015). Long noncoding RNA PVT1 indicates a poor prognosis of gastric cancer and promotes cell proliferation through epigenetically regulating p15 and p16. Molecular Cancer, 14(1), 82. http://dx.doi. org/10.1186/s12943-015-0355-8. PMid:25890171.

Liu, Z., Yan, Y., Cao, S., \& Chen, Y. (2018). Long non-coding RNA SNHG14 contributes to gastric cancer development through targeting miR-145/SOX9 axis. Journal of Cellular Biochemistry, 119(8), 69056913. http://dx.doi.org/10.1002/jcb.26889. PMid:29667771.

Ma, Z., Gu, S., Song, M., Yan, C., Hui, B., Ji, H., Wang, J., Zhang, J., Wang, K., \& Zhao, Q. (2017). Long non-coding RNA SNHG17 is an unfavourable prognostic factor and promotes cell proliferation by epigenetically silencing P57 in colorectal cancer. Molecular BioSystems, 13(11), 2350-2361. http://dx.doi.org/10.1039/C7MB00280G. PMid:28933484.

Rafiq, S., Gulzar, N., Huma, N., Hussain, I., \& Murtaza, M. S. (2020). Evaluation of anti-proliferative activity of Cheddar cheeses using colon adenocarcinoma (HCT-116) cell line. International Journal of Dairy Technology, 73(1), 255-260. http://dx.doi.org/10.1111/14710307.12665 .

Thiel, A., \& Ristimäki, A. (2012). Gastric cancer: basic aspects. Helicobacter, 17(Suppl. 1), 26-29. http://dx.doi.org/10.1111/j.15235378.2012.00979.x. PMid:22958152.

Xie, M., Sun, M., Zhu, Y. N., Xia, R., Liu, Y. W., Ding, J., Ma, H. W., He, X. Z., Zhang, Z. H., Liu, Z. J., Liu, X. H., \& De, W. (2015). Long noncoding RNA HOXA-AS2 promotes gastric cancer proliferation by epigenetically silencing P21/PLK3/DDIT3 expression. Oncotarget, 6(32), 33587-33601. http://dx.doi.org/10.18632/oncotarget.5599. PMid:26384350.

Ye, F., Gong, Y., Chen, X., Yu, M., Zuo, Z., Pei, D., Liu, W., Wang, Q., Zhou, J., Duan, L., Zhang, L., Li, X., Tang, T., \& Huang, J. (2018). Long noncoding AFAP1-antisense RNA 1 is upregulated and promotes tumorigenesis in gastric cancer. Oncology Letters, 15(5), 7523-7530. http://dx.doi.org/10.3892/ol.2018.8266. PMid:29740481.

Yu, J., Tian, X., Chang, J., Liu, P., \& Zhang, R. (2017). RUNX3 inhibits the proliferation and metastasis of gastric cancer through regulating miR-182/HOXA9. Biomedicine and Pharmacotherapy, 96, 782-791. http://dx.doi.org/10.1016/j.biopha.2017.08.144. PMid:29054094.

Zhang, M., \& Du, X. (2016). Noncoding RNAs in gastric cancer: research progress and prospects. World Journal of Gastroenterology, 22(29), 6610-6618. http://dx.doi.org/10.3748/wjg.v22.i29.6610. PMid:27547004.

Zhang, X., Liang, W., Liu, J., Zang, X., Gu, J., Pan, L., Shi, H., Fu, M., Huang, Z., Zhang, Y., Qian, H., Jiang, P., \& Xu, W. (2018a). Long non-coding RNA UFC1 promotes gastric cancer progression by regulating miR-498/Lin28b. Journal of Experimental \& Clinical Cancer Research, 37(1), 134. http://dx.doi.org/10.1186/s13046-0180803-6. PMid:29970131.

Zhang, Y., Yu, S., Zhang, Z., Zhao, G., \& Xu, J. (2018b). Long noncoding RNA AK096174 promotes cell proliferation and invasion in gastric cancer by regulating WDR66 expression. Bioscience Reports, 38(4), BSR20180277. http://dx.doi.org/10.1042/BSR20180277. PMid:29717028.

Zhao, H., Zhang, K., Wang, T., Cui, J., Xi, H., Wang, Y., Song, Y., Zhao, X., Wei, B., \& Chen, L. (2018). Long non-coding RNA AFAP1-antisense RNA 1 promotes the proliferation, migration and invasion of gastric cancer cells and is associated with poor patient survival. Oncology Letters, 15(6), 8620-8626. http://dx.doi.org/10.3892/ol.2018.8389. PMid:29805596. 